\title{
Hidden GeV-scale interactions of quarks
}

\author{
Bogdan A. Dobrescu and Claudia Frugiuele \\ Theoretical Physics Department, Fermilab, Batavia, Illinois, USA
}

(Dated: April 24, 2014)

\begin{abstract}
We explore quark interactions mediated by new gauge bosons of masses in the $0.3-50 \mathrm{GeV}$ range. A tight upper limit on the gauge coupling of light $Z^{\prime}$ bosons is imposed by the anomaly cancellation conditions in conjunction with collider bounds on new charged fermions. Limits from quarkonium decays are model dependent, while electroweak constraints are mild. We derive the limits for a $Z^{\prime}$ boson coupled to baryon number, and then construct a $Z^{\prime}$ model with relaxed constraints, allowing quark couplings as large as 0.2 for a mass of a few $\mathrm{GeV}$.
\end{abstract}

Introduction.-Quarks experience all five known interactions: strong, weak, electromagnetic, Higgs and gravitational. It behooves us to ask whether additional interactions of quarks exist, and what are the current limits on their strength. Experimental searches for new particles interacting with quarks have been performed at hadron colliders over the last few decades, setting upper limits on their couplings for masses in the $50 \mathrm{GeV}-3 \mathrm{TeV}$ range [1, 2]. Smaller masses have been less intensely investigated, due to large backgrounds at hadron colliders.

Since the quarks are fermions, the mediators of quark interactions must be bosons. Restricting attention to renormalizable quantum field theories, elementary mediators can have spin 0 or 1 . Due to the chiral nature of the known quarks, the light quarks may have only suppressed interactions with spin-0 mediators. It is less clear whether large couplings may be allowed for spin-1 mediators. In this letter we study existing limits on the coupling of new spin-1 particles that interact with quarks, and have masses in the $0.3-50 \mathrm{GeV}$ range. Lighter mediators are possible, but their couplings are strongly constrained [3], and precise limits are harder to derive for masses near the QCD scale.

Spin-1 fields are well behaved at high energies only if they are gauge bosons. While composite spin-1 particles bound by some new dynamics may exist, their coupling to quarks would suggest a compositeness scale above the weak scale $(v \approx 246 \mathrm{GeV})$; given that spin- 1 states are typically near the compositeness scale, we focus on elementary gauge bosons. Only electrically-neutral gauge bosons with highly suppressed couplings to leptons ("leptophobic") are allowed at masses below $50 \mathrm{GeV}$. These can be color singlets (i.e., $Z^{\prime}$ bosons) or octets. The latter are severely constrained by the running of the QCD coupling below $M_{Z}$ [4]. Thus, leptophobic $Z^{\prime}$ bosons associated with a $U(1)_{z}$ gauge extension of the Standard Model (SM) are the best candidates for mediating new relatively-strong quark interactions at low energies.

$Z^{\prime}$ couplings to quarks. - The renormalizable interactions of a $Z^{\prime}$ boson of this type are given by

$$
\frac{g_{z}}{2} Z_{\mu}^{\prime}\left(z_{Q_{j}} \bar{Q}_{L}^{j} \gamma^{\mu} Q_{L}^{j}+z_{u_{j}} \bar{u}_{R}^{j} \gamma^{\mu} u_{R}^{j}+z_{d_{j}} \bar{d}_{R}^{j} \gamma^{\mu} d_{R}^{j}\right),
$$

where $j$ labels the generations, $Q_{L}^{j}$ are the left-handed quark doublets, $u_{R}^{j}, d_{R}^{j}$ are the right-handed quark gauge eigenstates, $z_{Q_{j}}, z_{u_{j}}, z_{d_{j}}$ are their $U(1)_{z}$ charges, and $g_{z}$ is the gauge coupling. Higher-dimensional $Z^{\prime}$ interactions may exist [5], but their effects for a $Z^{\prime}$ mass $M_{Z^{\prime}} \ll v$ are suppressed.

A simple charge assignment that allows quark masses and evades constraints from flavor-changing neutral currents (FCNC) is $z_{Q_{j}}=z_{u_{j}}=z_{d_{j}}=1 / 3$, i.e., charges given by the baryon number. Experimental limits on $g_{z}$ are loose in this case [6, 7], except for $M_{Z^{\prime}}$ near the $\Upsilon$ or $J / \psi$ resonances. We will show, however, that additional limits on light leptophobic $Z^{\prime}$ bosons arise from the interplay of collider limits on new fermions and theoretical constraints.

Anomaly cancellation.-Self-consistency of the theory at high energies [8] requires gauge anomaly cancellation. Given that $U(1)_{z}$ must be embedded at some high scale in a non-Abelian gauge group, the charges should be commensurate. Thus, for certain $g_{z}$ normalizations, the $U(1)_{z}$ charges are integers, so that finding solutions to the $\left[U(1)_{z}\right]^{3}$ anomaly cancellation is non-trivial.

Even without fermions beyond the SM, leptophobic $U(1)_{z}$ groups can be anomaly free, e.g., when first (second) generation quarks have $U(1)_{z}$ charge $z_{1}\left(z_{2}\right)$, and third-generation quarks have charge $-\left(z_{1}+z_{2}\right)$. For $M_{Z}^{\prime} \ll v$, though, $Z^{\prime}$-induced FCNC are large unless the $Q_{L}^{j}$ charges are equal $\left(u_{R}^{j}\right.$ or $d_{R}^{j}$ may have $j$-dependent charges because their gauge and mass eigenstates may be identical): $K^{0}-\bar{K}^{0}$ mixing requires $g_{z}\left|z_{Q_{3}}-z_{Q_{1}}\right|<$ $10^{-5} M_{Z^{\prime}} /(1 \mathrm{GeV})$, and $B^{0}-\bar{B}^{0}$ mixing imposes a slightly weaker constraint on $g_{z}\left|z_{Q_{3}}-z_{Q_{1}}\right|$. Thus, large values of $g_{z}$ require $z_{Q_{1}}=z_{Q_{2}}=z_{Q_{3}}$. Then, in the absence of new fermions, the $\left[S U(2)_{W}\right]^{2} U(1)_{z}$ anomaly implies $z_{Q_{j}}=0$. The remaining anomaly cancellations [9] imply $z_{u_{3}}=-z_{u_{1}}-z_{u_{2}}$ and $z_{d_{3}}=-z_{d_{1}}-z_{d_{2}}$, as well as

$$
\begin{gathered}
2\left(z_{u_{1}}^{2}+z_{u_{2}}^{2}+z_{u_{1}} z_{u_{2}}\right)=z_{d_{1}}^{2}+z_{d_{2}}^{2}+z_{d_{1}} z_{d_{2}}, \\
-z_{u_{1}} z_{u_{2}}\left(z_{u_{1}}+z_{u_{2}}\right)=z_{d_{1}} z_{d_{2}}\left(z_{d_{1}}+z_{d_{2}}\right) .
\end{gathered}
$$

A necessary condition for these equations to have integer solutions is that there exist an integer $k$ such that

$$
z_{u_{1}}^{3}-6 z_{u_{1}}^{2} z_{d_{1}}+4 z_{d_{1}}^{3}=k^{2}\left(z_{u_{1}}+2 z_{d_{1}}\right) .
$$

We have checked numerically that this condition is not satisfied for $\left|z_{u_{1}}\right|,\left|z_{d_{1}}\right| \leq 1000$. For practical purposes, thus, anomaly-free solutions with generationindependent $z_{Q_{j}}$ require fermions beyond the SM. 
A fourth generation of chiral fermions is ruled out by direct searches for new quarks at the LHC [10]. Anomalyfree sets of color-singlet chiral fermions [11] are severely constrained by $h^{0} \rightarrow \gamma \gamma$ and electroweak measurements.

We are then lead to consider fermions that are vectorlike with respect to the SM gauge group, and chiral under $U(1)_{z}$. Among the new fermions required to cancel the various anomalies, there are electrically charged ones. If these are long-lived, then LHC searches for slowly ionizing charged tracks set a mass limit $m_{f}>450 \mathrm{GeV}$ (we have compared the experimental limit [12] with the pair-production cross section for a charge-1 lepton computed with MadGraph [13]). Decays of charged vectorlike fermions into neutral ones may relax the limits. The 1-loop mass splitting between the components $N^{0}$ and $E^{ \pm}$of a weak-doublet vector-like lepton is $\sim 0.3 \mathrm{GeV}$ [14]. For a stable $N^{0}$, the process $e^{+} e^{-} \rightarrow E^{+} E^{-}$leads to a final state with two soft pions and missing energy. The mass limit, using initial state radiation at LEP, is $\sim 90 \mathrm{GeV}$ [15]; for the future LHC reach, see [16].

Lower limits on vector-like fermion masses translate into an upper limit on $g_{z}$. Note that $M_{Z^{\prime}}=g_{z} z_{\varphi}\langle\varphi\rangle / 2$, where $\varphi$ is the scalar whose VEV breaks $U(1)_{z}$. A new fermion, $f$, that is chiral with respect to $U(1)_{z}$ acquires a mass $m_{f}=\lambda\langle\varphi\rangle$ via a Yukawa term $\lambda \varphi \bar{f}_{L} f_{R}$. Given that the Yukawa coupling blows up in the UV, there is a perturbativity limit $\lambda \lesssim 4 \pi / 3$; a lower limit on $m_{f}$ then implies a bound on $g_{z}$ :

$$
g_{z}=\frac{2 \lambda M_{Z^{\prime}}}{z_{\varphi} m_{f}} \lesssim \frac{8.4 \times 10^{-2}}{z_{\varphi}}\left(\frac{M_{Z^{\prime}}}{1 \mathrm{GeV}}\right)\left(\frac{100 \mathrm{GeV}}{m_{f}}\right) .
$$

For $U(1)_{z}$ charges given by the baryon number (we refer to this assignment as the $Z_{B}^{\prime}$ model), the $\left[S U(3)_{c}\right]^{2} U(1)_{z}$ anomaly cancels, so that all vector-like fermions may be color singlets [9, 17] (solutions with color triplets also exist [2]). To avoid a large $Z-Z^{\prime}$ mixing, the $U(1)_{Y}$ and $U(1)_{z}$ charges must satisfy $\operatorname{Tr}(z Y)=0$. The minimal set of vector-like fermions consists of a weak doublet $\left(Y=-1 / 2, z_{L}=-1, z_{R}=+2\right)$, a weak singlet $(Y=-1$, $\left.z_{L}=+2, z_{R}=-1\right)$, and a SM singlet $\left(z_{L}=+2, z_{R}=-1\right.$; or $\left.z_{L}=+1, z_{R}=-2\right)$; their masses require $z_{\varphi}=3$. If the charged fermions are slightly heavier than the neutral ones, then the collider signal is again soft pions and missing energy so that $m_{f}>90 \mathrm{GeV}$, leading to the $g_{z}$ limit given by the middle straight line in Fig. 1. Without tuning, though, the mass splittings are large, the collider limits on $m_{f}$ are higher, and the upper limit on $g_{z}$ decreases (see, e.g., the $m_{f}=450 \mathrm{GeV}$ line in Fig. 1).

A loophole is that several $\varphi$ scalars may break $U(1)_{z}$. If $n$ scalars have equal VEVs and equal charges, the $g_{z}$ limit is relaxed by a factor of $\sqrt{n}$. Plausible theories, though, do not have $n$ larger than a few. Another loophole is that there can be $N_{f}$ copies of the minimal vector-like fermion set with $U(1)_{z}$ charges smaller by a factor of $N_{f}$, implying $z_{\varphi}=3 / N_{f}$ and a $g_{z}$ limit increased by $N_{f}$ (see the $N_{f}=3, m_{f}=90 \mathrm{GeV}$ line in Fig. 1).

Promptly decaying vector-like fermions. - Let us explore whether the mass limits can be relaxed when the

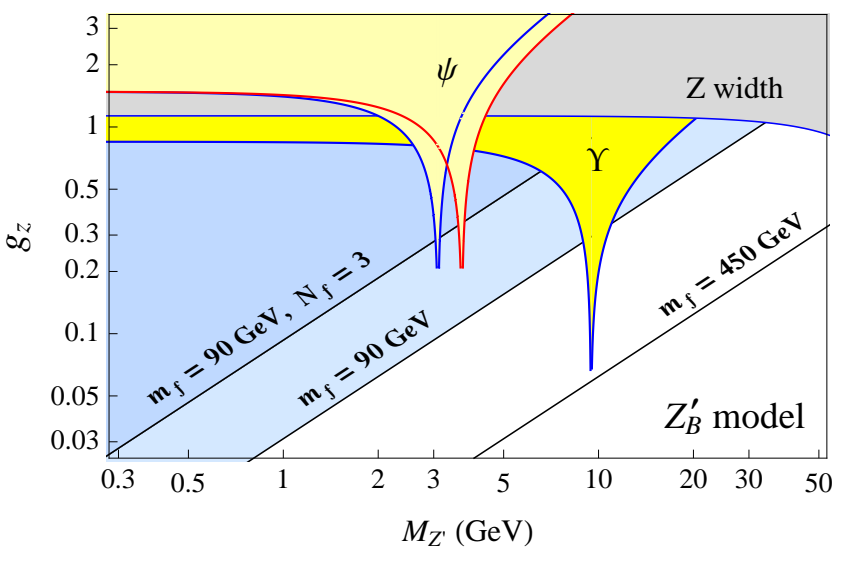

FIG. 1. Limits in the gauge coupling versus mass plane for $Z_{B}^{\prime}$. Values of $g_{z}$ above the straight lines are excluded by the anomaly cancellation conditions in conjunction with collider searches for new fermions, for $N_{f}=1$ or $3, m_{f}>90$ $\mathrm{GeV}$, and for $N_{f}=1, m_{f}>450 \mathrm{GeV}$. The top regions are excluded by quarkonium and hadronic $Z$ decays.

vector-like fermions decay into SM particles. A vectorlike fermion may decay through mixing with a SM one if they couple to $\varphi$. The new mass-eigenstate fermion has 4 decay channels, into a SM fermion and one of the heavy bosons, $W, Z, h^{0}$ or $Z^{\prime}$. The branching fraction involving $Z^{\prime}$ is typically small, of order $\left(g_{z} z_{f} / g\right)^{2} / 4 \ll 1$, where $z_{f}$ is the $U(1)_{z}$ charge of the mixed component of the new fermion, and $g \approx 0.65$ is the weak gauge coupling. The LHC limits on vector-like quarks that decay into a SM quark and a $W, Z$, or $h^{0}$ boson are stringent, above $700 \mathrm{GeV}$ [10]. Vector-like leptons are less constrained. The LHC limits on processes involving weak bosons and missing transverse energy or charged leptons have been recast as mass limits on vector-like leptons [18]. Weakdoublet leptons must be heavier than about $280 \mathrm{GeV}$ if they decay to $\tau Z$ and $\tau W$ (the limit is $460 \mathrm{GeV}$ if the $\tau$ is replaced by an $e$ or $\mu$ ).

If the SM quark doublets are $U(1)_{z}$-neutral, the vectorlike fermions can be weak singlets, so that production at the LHC cannot proceed through a $W$, and the $g_{z}$ limit is relaxed. Weak-singlet charged leptons decaying into $e Z$ or $\mu Z$ must be heavier than $\sim 100 \mathrm{GeV}$, while no LHC limit can be derived for the $\tau Z$ decay [18]. The LEP mass limit on leptons decaying into $\nu W$ is $101 \mathrm{GeV}$ [19].

Vector-like fermions lighter than $W$ would decay predominantly into a SM fermion and a $Z^{\prime}$, which in turn decays into two jets. Pair production of a vector-like quark decaying into a light quark and a $Z^{\prime}$ leads to a 6-jet final state. Masses between $77 \mathrm{GeV}$ and $\sim 100 \mathrm{GeV}$ are ruled out by a CDF search [20], provided that $M_{Z}^{\prime}$ is not so small that the jets overlap. Masses below $M_{Z} / 2$ are excluded by measurements of hadronic $Z$ decays. In the $46-77 \mathrm{GeV}$ mass range there is no limit for a quark decaying into three jets; although this gap should be explored by future searches, we do not discuss it further.

New physics could lead to decays of vector-like fermions that are harder to detect. If the new fermions 
do not mix with SM ones, and there are 4-fermion operators (induced, e.g., by a very heavy boson), then the vector-like fermions can decay predominantly into three SM fermions. These can all be light quarks if the vectorlike fermion is a color triplet. The $6 j \mathrm{CDF}$ search has set a mass limit on gluinos of $140 \mathrm{GeV}$, but the cross section for quark pair-production is smaller by a factor of about 3 , so that the lower mass limit on vector-like quarks is only $100 \mathrm{GeV}$. Similarly, there are no limits on vector-like quarks from existing $6 j$ CMS searches [21].

Vector-like leptons might partially evade various collider searches if they decay into $\tau j j$. A LEP search for a pair of $\tau j$ resonances sets a mass limit of $98 \mathrm{GeV}$ on leptoquarks [22]; the extra jet from the vector-like lepton decay would relax this limit, but it seems unlikely that it would be pushed below $\sim 90 \mathrm{GeV}$.

Quarkonium decays. - Searches for nonstandard $\Upsilon$ decays constrain the $Z^{\prime}$ couplings to $b$ quarks. The ratio of branching fractions $\Delta R_{\Upsilon} \equiv B\left(\Upsilon \rightarrow Z^{\prime *}, \gamma^{*} \rightarrow j j\right) / B(\Upsilon \rightarrow$ $\left.\mu^{+} \mu^{-}\right)$can be used [6, 7] to set limits on $g_{z}$. The limit on the non-electromagnetic di-jet decay of $\Upsilon(1 S)$ [23], $\Delta R_{\Upsilon}<2.1$, gives the excluded region shown in Fig. 1 (labelled " $\Upsilon$ ") for the $Z_{B}^{\prime}$ model.

The axial $Z^{\prime}$ coupling to $b$ quarks is constrained for masses below $7 \mathrm{GeV}$ by the process $\Upsilon \rightarrow \gamma Z^{\prime}$. The search [24] for $\Upsilon(2 S) \rightarrow \gamma A^{0}$ where $A^{0}$ is a pseudo-scalar decaying into hadrons, set a branching fraction limit of $10^{-6}$ at $M_{A^{0}}=1 \mathrm{GeV}$. A similar limit applies to $\Upsilon \rightarrow \gamma Z^{\prime}$, with the difference arising from the acceptance, which depends on the spin. This does not affect $Z^{\prime}$ s that have only a vector coupling, such as $Z_{B}^{\prime}$.

Charmonium decays into hadrons set limits on the $Z^{\prime}$ couplings to $c$ quarks. We focus on exclusive $J / \psi$ and $\psi(2 S)$ decays into $K^{+} K^{-}$. The photon contribution appears to saturate the measured branching fractions, although there are uncertainties from interference with QCD effects [25]. The ratio $r_{\psi} \equiv B\left(\psi \rightarrow Z^{\prime *}, \gamma^{*} \rightarrow\right.$ $\left.K^{+} K^{-}\right) / B\left(\psi \rightarrow \gamma^{*} \rightarrow K^{+} K^{-}\right)$is then bounded on both sides: $1 / 2 \sim r_{\psi}^{\min }<r_{\psi}<r_{\psi}^{\max } \sim 2$. Computing the $Z^{\prime}$ and $\gamma$ contributions in the $Z_{B}^{\prime}$ model we obtain

$$
g_{z}^{2} \lesssim \frac{24 e^{2}}{\varepsilon_{s}}\left(1-\sqrt{r_{\psi}^{\mathrm{m}}}\right)\left(1-\frac{M_{Z^{\prime}}^{2}}{M_{\psi}^{2}}\right)
$$

where $r_{\psi}^{\mathrm{m}}=r_{\psi}^{\min }$ for $M_{Z^{\prime}}<M_{\psi}$, and $r_{\psi}^{\mathrm{m}}=r_{\psi}^{\max }$ for $M_{Z^{\prime}}>M_{\psi} ; \varepsilon_{s} \approx m_{s} / \Lambda_{\mathrm{QCD}}$ parametrizes the flavor $S U(3)$ violation, and $e$ is the electromagnetic gauge coupling. The excluded regions are shown in Fig. 1 (labelled " $\psi$ ") for $\varepsilon_{s}=1 / 3$, with excisions at $\left|M_{Z}^{\prime} / M_{\psi}-1\right|<10^{-2}$ where the mixing between $\psi$ and $Z^{\prime}$ is large. The constraints from inclusive decays are estimated in [7]. In models with $z_{u} \neq z_{d}$ there are stronger constraints from $\psi \rightarrow Z^{*} \rightarrow \pi^{+} \pi^{-}$.

Similarly, the $Z^{\prime}$ couplings to $s$ quarks contribute to $\phi$ meson decays into $\pi^{+} \pi^{-}$, where photon exchange dominates [26]. In the $Z_{B}^{\prime}$ model the effect violates isospin so it is suppressed by $\left(m_{d}-m_{u}\right) / \Lambda_{\mathrm{QCD}}$, and the limits are not competitive (while they are stringent for $z_{u} \neq z_{d}$ ).

Electroweak observables.-As long as $\operatorname{Tr}(z Y)=0$, the 1-loop kinetic mixings of $Z^{\prime}$ with $Z\left(c_{Z}\right)$ and $\gamma$ lead only to mild constraints. The largest effect identified in [6, [7] is a change in the hadronic $Z$ width. The $Z_{B}^{\prime}$ model with $g_{z}$ normalized as in Eq. (11) gives $c_{Z} \approx 0.01 g_{z}$ [27] and

$$
\frac{\Delta \Gamma_{Z}^{\mathrm{had}}}{\Gamma_{Z}^{\mathrm{had}}}=\frac{2 g_{z} c_{Z} c_{W} s_{W}\left(2 V_{u}+3 V_{d}\right)}{3 g\left(1-M_{Z^{\prime}}^{2} / M_{Z}^{2}\right)\left(2 V_{u}^{2}+3 V_{d}^{2}+5 / 16\right)},
$$

where $V_{u, d}= \pm 1 / 4-(3 \pm 1) s_{W}^{2} / 6$, and $s_{W} \equiv \sin \theta_{W}$. This rules out the region labelled " $Z$ width" in Fig. 1.

Down-strange $Z^{\prime}$ at the GeV scale.-The bounds from $\Upsilon$ and $J / \psi$ decays are avoided if the $Z^{\prime}$ couplings to $b$ and $c$ quarks vanish. This is consistent with quark mass generation, without inducing tree-level FCNC, when only right-handed quarks carry $U(1)_{z}$ charges. If $d_{R}$ and $s_{R}$ have opposite charges $\left(z_{d_{1}}=-z_{d_{2}}\right)$ and all other SM fields are $U(1)_{z}$ neutral (we refer to this assignment as the $Z_{d s}^{\prime}$ model), then the only anomaly that remains to be cancelled is $U(1)_{Y}\left[U(1)_{z}\right]^{2}$. A set of vector-like fermions

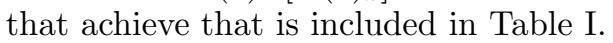

Tree-level FCNC are absent provided the gauge and mass eigenstates coincide for right-handed down-type quarks. Let us outline a mechanism for quark mass generation that satisfies this condition. The $b$ quark acquires mass from a SM Yukawa term $y_{b} H \bar{b}_{R} Q_{L}^{3}$, where $H$ is the Higgs doublet. We have chosen a basis where the Yukawa couplings of $Q_{L}^{1}$ and $Q_{L}^{2}$ to $b_{R}$ vanish. The $s$ and $d$ quark masses are generated by dimension- 5 operators:

$$
\frac{c_{j}^{s}}{m_{1}^{\omega}} \varphi^{\dagger} H \bar{s}_{R} Q_{L}^{j}+\frac{c_{j}^{d}}{m_{2}^{\omega}} \varphi H \bar{d}_{R} Q_{L}^{j}+\text { H.c. , }
$$

where $m_{1}^{\omega}, m_{2}^{\omega}$ are mass parameters, and $c_{j}^{s}, c_{j}^{d}$ are dimensionless coefficients. Without loss of generality we take $c_{1}^{s}=0$, so that $m_{1}^{\omega}$ is related to the strange quark mass by $m_{1}^{\omega}=c_{2}^{s} v M_{Z^{\prime}} /\left(g_{z} m_{s}\right)$. For $c_{2}^{s} \lesssim O(1)$ we find $m_{1}^{\omega} \lesssim 2.5 \mathrm{TeV} / g_{z}$ for $M_{Z^{\prime}} \approx 1 \mathrm{GeV}$, which shows that the mass scale where the strange quark mass is generated may be explored at the LHC.

A renormalizable origin of the operators (7) is provided by two vector-like quarks, $\omega^{i}, i=1,2$, which transform as $b_{R}$ and have Yukawa couplings to the $d$ and $s$ quarks:

$$
y_{i j}^{\omega} H \bar{\omega}_{R}^{i} Q_{L}^{j}+\lambda_{i}^{s} \varphi^{\dagger} \bar{s}_{R} \omega_{L}^{i}+\lambda_{i}^{d} \varphi \bar{d}_{R} \omega_{L}^{i}+\text { H.c. }
$$

\begin{tabular}{c|ccc|c}
\hline field & $S U(3)_{C}$ & $S U(2)_{W}$ & $U(1)_{Y}$ & $U(1)_{z}$ \\
\hline$d_{R}, s_{R}$ & 3 & 1 & $-1 / 3$ & $+1,-1$ \\
$f_{L}, f_{L}^{\prime}$ & 1 & 1 & +1 & 0 \\
$f_{R}, f_{R}^{\prime}$ & 1 & 1 & +1 & $+1,-1$ \\
\hline$\varphi$ & 1 & 1 & 0 & +1 \\
\hline
\end{tabular}

TABLE I. Fields carrying $U(1)_{z}$ charge in the $Z_{d s}^{\prime}$ model. 


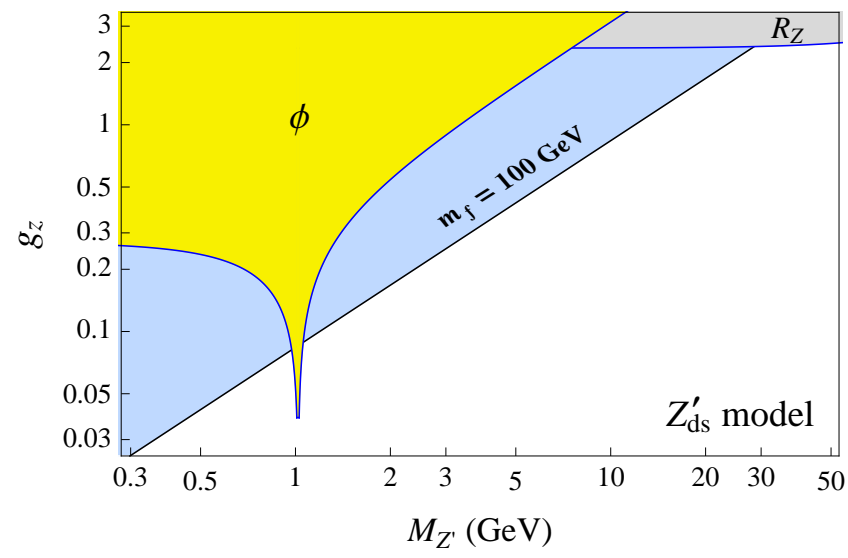

FIG. 2. Limits in the $g_{z}$ versus $M_{Z^{\prime}}$ plane for $Z_{d s}^{\prime}$. The shaded regions are excluded (see caption to Fig 1 ).

An $U(2)_{L}$ transformation of $Q_{L}^{1}$ and $Q_{L}^{2}$ can set $y_{21}^{\omega}=0$. Similarly, the $\bar{b}_{R} \omega_{L}^{i}$ terms can be rotated away, and the vector-like quark masses can be diagonalized, $m_{i}^{\omega} \bar{\omega}^{i} \omega^{i}$. Comparing the operators (7) with the above Yukawa terms, we find $c_{i}^{s} \simeq y_{1 i}^{\omega}$ and $c_{i}^{d} \simeq y_{2 i}^{\omega} \lambda_{2}^{d}$ for $m_{1}^{\omega} \ll m_{2}^{\omega}$.

Mass terms involving $f_{L}$ or $f_{L}^{\prime}$ (see Table I) and righthanded SM leptons can be kept small by an approximate discrete symmetry. These terms induce mixing of the vector-like fermions with the SM leptons, so that $f$ and $f^{\prime}$ decay predominantly into $W \nu$. The LEP bound on $f$ and $f^{\prime}$ masses is then around $100 \mathrm{GeV}$. Assuming that the form factors for vector and axial couplings are equal, the $g_{z}$ limit from $\phi$ meson decay is given by Eq. (5) with $\varepsilon_{s} \rightarrow 9$ and $\psi \rightarrow \phi$, and is shown in Fig. 2. If $f$ and $f^{\prime}$ have equal mass, then the $Z-Z^{\prime}$ mixing is suppressed.
The change in hadronic $Z$ decays, due to $Z^{\prime}$ emission and 1-loop corrections, excludes the region labelled $R_{Z}$ in Fig. 2 (based on [6] with updated $\Delta \alpha_{s}$ ).

Additional constraints for $M_{Z}^{\prime} \lesssim 0.5 \mathrm{GeV}$ arise from meson decays such as $\eta \rightarrow \gamma Z^{\prime} \rightarrow \gamma \pi^{+} \pi^{-}$or $\eta^{\prime} \rightarrow Z^{\prime} Z^{\prime} \rightarrow$ $\pi^{+} \pi^{-} \pi^{+} \pi^{-}$. Currently, the limits are weaker than the ones shown in Fig. 2, but future searches in these channels for $\pi^{+} \pi^{-}$resonances may probe lower values of $g_{z}$.

Conclusions. - Besides the usual limits on light leptophobic $Z^{\prime}$ bosons, from $\Upsilon$ decays and electroweak observables, we have found a strong constraint from the requirement that new fermions cancel the gauge anomalies. Collider limits on the new fermion masses imply an upper limit on the gauge coupling. The same applies to other light gauge bosons with anomalous charges for SM fermions.

Nevertheless, the gauge coupling of a baryonic $Z^{\prime}$ may be relatively large, of order 0.1 for $M_{Z}^{\prime} \gtrsim 2 \mathrm{GeV}$ (Fig. 1). Furthermore, we have presented a renormalizable model where the only SM fields charged under the new group are $d_{R}$ and $s_{R}$, allowing even larger couplings (Fig. 2).

Future experiments may search for $\mathrm{GeV}$-scale leptophobic $Z^{\prime}$ s in various ways, including non-standard meson decays and LHC signatures of boosted di-jet resonances, as well as test them through searches for vectorlike fermions. If the $Z^{\prime}$ also couples to light dark matter particles, then interesting phenomena may be uncovered in neutrino detectors [28] and other experiments.

Acknowledgments: We thank Prateek Agrawal, Andre de Gouvea, Andreas Kronfeld and Felix Yu, for constructive comments.
[1] J. Beringer et al. [Particle Data Group], "Review of Particle Physics", Phys. Rev. D 86, 010001 (2012).

[2] B. A. Dobrescu, F. Yu, "Coupling-mass mapping of dijet peak searches," Phys. Rev. D 88, 035021 (2013) arXiv:1306.2629.

[3] A. E. Nelson, N. Tetradis, "Constraints on a new vector boson coupled to baryons," Phys. Lett. B 221, 80 (1989);

[4] G. Z. Krnjaic, "Very light axigluons and the top asymmetry," Phys. Rev. D 85, 014030 (2012) arXiv:1109.0648.

[5] P. J. Fox et al., "An effective $Z^{\prime}$," Phys. Rev. D 84, 115006 (2011) arXiv:1104.4127.

[6] C. D. Carone, H. Murayama, "Possible light $U(1)$ gauge boson coupled to baryon number," Phys. Rev. Lett. 74, 3122 (1995) hep-ph/9411256; "Realistic models with a light $U(1)$ gauge boson coupled to baryon number," Phys. Rev. D 52, 484 (1995) hep-ph/9501220; D. C. Bailey, S. Davidson, "Is there a vector boson coupling to baryon number?", Phys. Lett. B 348, 185 (1995) hep-ph/9411355.

[7] A. Aranda, C. D. Carone, "Limits on a light leptophobic gauge boson," Phys. Lett. B 443, 352 (1998) hep-ph/9809522.

[8] W. A. Bardeen, "Anomalous Ward identities in spinor field theories," Phys. Rev. 184, 1848 (1969).

[9] M. S. Carena, et al., " $Z$ ' gauge bosons at the Tevatron," Phys. Rev. D 70, 093009 (2004) hep-ph/0408098.

[10] S. Chatrchyan et al. [CMS Collaboration], "Inclusive search for a vector-like $T$ quark with charge $2 / 3$ ", Phys. Lett. B 729, 149 (2014) arXiv:1311.7667; "Combined search for the quarks of a sequential fourth generation," Phys. Rev. D 86, 112003 (2012) arXiv:1209.1062.

[11] A. de Gouvea, W. -C. Huang, J. Kile, "Dark matter from weak polyplets," arXiv:1207.0510

[12] ATLAS Collaboration, "A search for heavy long-lived sleptons using $16 \mathrm{fb}^{-1}$ of $p p$ collisions", note CONF-2013058, Aug. 2013.

[13] J. Alwall, et al., "MadGraph 5: Going Beyond," JHEP 1106, 128 (2011) arXiv:1106.0522.

[14] S. D. Thomas, J. D. Wells, "Phenomenology of massive vectorlike doublet leptons," Phys. Rev. Lett. 81, 34 (1998) hep-ph/9804359]; S. Dimopoulos, et al. "TeV dark matter", Nucl. Phys. B 349, 714 (1991) [Erratumibid. B 357, 308 (1991)].

[15] A. Heister et al. [ALEPH Collaboration], "Search for charginos nearly mass degenerate with the lightest neutralino", Phys. Lett. B 533, 223 (2002) hep-ex/0203020]. 
[16] J. Halverson, N. Orlofsky, A. Pierce, "Vectorlike leptons as the tip of the dark matter iceberg," arXiv:1403.1592.

[17] M. Duerr, P. Fileviez Perez, M. B. Wise, "Gauge theory for baryon and lepton numbers with leptoquarks," Phys. Rev. Lett. 110, 231801 (2013) arXiv:1304.0576.

[18] A. Falkowski, D.M. Straub, A. Vicente, "Vector-like leptons: Higgs decays and collider phenomenology", arXiv:1312.5329.

[19] P. Achard et al. [L3 Collaboration], "Search for heavy neutral and charged leptons in $e^{+} e^{-}$annihilation at LEP," Phys. Lett. B 517, 75 (2001) hep-ex/0107015.

[20] T. Aaltonen et al. [CDF Collaboration], "First search for multijet resonances in $\sqrt{s}=1.96 \mathrm{TeV} p \bar{p}$ collisions," Phys. Rev. Lett. 107, 042001 (2011) arXiv:1105.2815.

[21] S. Chatrchyan et al. [CMS Collaboration], "Searches for light- and heavy-flavour three-jet resonances at $\sqrt{s}=8$ TeV," Phys. Lett. B 730, 193 (2014) arXiv:1311.1799; "Search for three-jet resonances at $\sqrt{s}=7 \mathrm{TeV}$," Phys. Lett. B 718, 329 (2012) arXiv:1208.2931.

[22] G. Abbiendi et al. [OPAL Collaboration], "Search for pair produced leptoquarks in $e^{+} e^{-}$interactions", Eur. Phys. J. C 31, 281 (2003) hep-ex/0305053.

[23] H. Albrecht et al. [ARGUS Collaboration], "An upper limit for two jet production in direct $\Upsilon(1 s)$ decays," Z . Phys. C 31, 181 (1986).

[24] J. P. Lees et al. [BaBar Collaboration], "Search for hadronic decays of a light Higgs boson in the radiative decay $\Upsilon \rightarrow \gamma A^{0}$," Phys. Rev. Lett. 107, 221803 (2011) arXiv:1108.3549.

[25] H. Czyz and J. H. Kuhn, "Strong and electromagnetic $J / \psi$ and $\psi(2 S)$ decays into pion and kaon pairs," Phys. Rev. D 80, 034035 (2009); arXiv:0904.0515.

[26] J. A. Oller, E. Oset, J. R. Pelaez, "The $\phi \rightarrow \pi^{+} \pi^{-}$decay within a chiral unitary approach," Phys. Rev. D 62, 114017 (2000) hep-ph/9911297.

[27] M. L. Graesser, I. M. Shoemaker, L. Vecchi, "A dark force for baryons," arXiv:1107.2666.

[28] B. Batell, M. Pospelov, A. Ritz, "Exploring portals to a hidden sector through fixed targets," Phys. Rev. D 80, 095024 (2009) arXiv:0906.5614. 